\title{
ABANDONO DE LOS ESTUDIOS UNIVERSITARIOS: DIMENSIÓN, FACTORES ASOCIADOS Y DESAFÍOS PARA LA POLITICA PÚBLICA
}

\author{
UNIVERSITY DROPOUT: \\ DIMENSIONS, DETERMINANTS AND CHALLENGES TO PUBLIC POLICY
}

Ana García de Fanelli Consejo Nacional de Investigaciones Científicas y Técnicas

Cecilia Adrogué de Deane Universidad Austral

Fecha de recepción: 04/03/2015

Fecha de aceptación: 25/06/2015

\section{RESUMEN}

Argentina se destaca en América latina por tener una de las tasas brutas de educación superior más altas, similar a la de los países desarrollados, fruto de un sistema no selectivo de admisión. No obstante, la igualdad de oportunidades en el acceso no ha sido suficiente para garantizar la equidad en los resultados. Teniendo como fuente de información la Encuesta Permanente de Hogares, en este artículo calculamos, en primer lugar, la tasa de abandono global total y según el nivel socioeconómico del hogar, el género y la región de residencia. En segundo lugar, estudiamos los factores asociados con la probabilidad de abandonar los estudios empleando un modelo de regresión logística. Concluimos que en el 201338 por ciento de los jóvenes que tenían al momento de la encuesta entre 18 y 30 años abandonaron la educación universitaria. Esta tasa presenta brechas significativas según nivel socioeconómico, género y región de residencia. Encontramos además que la probabilidad de abandonar es mayor entre los varones, los que trabajan, los que habitan en hogares de menor ingreso per cápita familiar y clima educativo y varía además según la región de residencia. El artículo concluye con una reflexión sobre las políticas públicas que podrían contribuir con la mejora en la persistencia y la equidad de resultados en el sector universitario.

Palabras clave: abandono de estudios, universidad, educación superior, equidad educativa

\section{ABSTRACT}

Argentina stands out in Latin America for having one of the foremost gross enrollment rates in higher education, similar to developed countries, as a consequence of an open-admissions system. However, equal opportunities in access alone do not ensure equity in the results. Using data from the Argentine Permanent Household Survey, we first calculate the global dropout rate according to the socioeconomic status of students' household, gender and area of residence. Second, we analyze the determinants of university students' propensity to drop out, estimating a logistic regression model. We conclude that in 2013,38 percent of those university students 
Abandono de los estudios universitarios: dimensión, factores asociados y desafíos para la política pública

between ages 18 and 30 dropped out of college. The data also revealed important gaps according to the socioeconomic status, the gender and area of residence. The probability of leaving college is higher among men, depends on whether they work and live in households with the lowest per capita income and educational level, and varies according to their area of residence. We conclude with some reflections on public policies that could contribute to improving the persistence and equity results in the Argentine university sector.

Key words: dropout, university, higher education, educational equity

\section{INTRODUCCIÓN}

Como lo muestra el Informe 2011 Educación Superior en Iberoamérica, la matrícula de educación superior se ha expandido en el espacio iberoamericano de un modo asombroso, pasando de 1.9 millones de estudiantes en 1970 a 22 millones en el 2008 (Brunner y Ferrada Hurtado, 2011). La Argentina, junto con España, Cuba, Venezuela y Uruguay, presenta un nivel de masificación alto que la ubica en el estadio de universalización de los estudios de educación superior según la tipología de Trow (2007).

En particular, la Argentina se destaca en América latina por tener una de las tasas brutas de educación superior más altas, 75,8 por ciento de la población entre los 20 y los 24 años en el 2011 (Ministerio de Educación, 2014). Este grado de escolarización es producto de mecanismos de admisión no selectivos en la mayoría de las instituciones', la fuerte regionalización de la oferta que ha tenido lugar en las últimas dos décadas tras la creación de numerosas universidades públicas y privadas en distintas zonas del país y la gratuidad de los estudios de grado (García de Fanelli, 2014a). No obstante, la ausencia de barreras académicas y económicas en la admisión a los estudios universitarios no garantiza la equidad de resultados debido a las brechas en términos de capital cultural, social y económico que presentan los estudiantes que egresan del nivel medio. Como lo señalaban Bourdieu y Passeron (2004:37) refiriéndose al caso francés pero aplicable también al argentino: "Difiriendo por completo en un conjunto de predisposiciones y pre-saberes que deben a su medio, los estudiantes sólo son iguales formalmente a la hora de la adquisición de la cultura académica".

\footnotetext{
1 El carácter no selectivo en el acceso como estudiante al sector universitario nacional se expresa en un proceso de admisión cuyo único requisito suele ser el poseer título de nivel medio y en la ausencia de un número máximo de estudiantes a admitir por carrera, es decir el establecimiento de vacantes o cupos. Empero, varias universidades han incorporado cursos de ingreso o de apoyo y nivelación no eliminatorios, cuya aprobación es necesaria para continuar los estudios de grado y cuyo fin es esencialmente mejorar la articulación entre la escuela media y la universidad.
} 
Esta desigualdad inicial de saberes y recursos y otros factores de la organización académica explican que, si bien en cuanto a matrícula la Argentina se encuentra muy bien posicionada, la proporción de graduados de nivel superior en la población adulta 2 era 16 por ciento en el 2010 frente al 33 por ciento en promedio en los países de la OECD (OECD, 2014). El punto de partida de nuestro estudio ha sido analizar el papel del abandono en el plano macro del sector universitario argentino ante esta situación compleja de alta escolarización y baja graduación.

En su clásico trabajo sobre al abandono universitario, el norteamericano Vicent Tinto (1975) señalaba que para estudiar este fenómeno es necesario distinguir entre el abandono definitivo de los estudios de nivel superior de aquel que tiene lugar en una institución de educación superior en particular o incluso en cierta carrera dentro de ésta. En un trabajo más reciente, Tinto (2012) introduce la distinción entre "retención" y "persistencia". El estudio de la retención hace referencia a la tasa a la cual una organización de educación superior retiene y gradúa después de un cierto número de años a aquellos estudiantes que se han incorporado a ella en un determinado momento del tiempo. El análisis de la persistencia, en cambio, presta atención a la acción del individuo de proseguir estudios de educación superior hasta su posterior graduación pero analizando esta cuestión desde el plano de la educación superior como un todo y no de una institución en particular. Lo que en este último caso interesa analizar es cuáles son los factores que en el plano sistémico inciden sobre la persistencia de los jóvenes en el nivel superior y eventualmente en su posterior graduación, contribuyendo así a la mejora del capital humano avanzado de un determinado país. Normalmente el nivel de abandono sistémico es mucho más bajo que el que tiene lugar en el plano de una organización particular de educación superior. Esto es así pues algunos estudiantes abandonan sus estudios en una cierta carrera o institución para continuarlos en otra disciplina o universidad o bien suspenden por un cierto tiempo por cuestiones familiares o laborales y luego prosiguen estudiando hasta graduarse. Estudios realizados en los Estados Unidos muestran que entre un tercio y la mitad de los estudiantes de las instituciones de educación superior de cuatro años de duración cambian de campo disciplinario al menos en una oportunidad mientras están estudiando para obtener el título de grado (Tinto, 2012).

\footnotetext{
2 Cálculo realizado en base a datos del Censo Nacional de Población y Vivienda 2010 (INDEC, 2012). En función de la agrupación de los datos publicados por tramos de edad, la población adulta comprendió a los que tenían entre 25 y 59 años en el año 2010.
} 
Dentro de la dicotomía, retención-abandono de una universidad o persistencia-abandono definitivo de los estudios de nivel superior, este artículo se ubica dentro de esta segunda perspectiva de análisis. En particular, examinamos el abandono que tiene lugar en el sector universitario en su conjunto, estudiando tanto la tasa global de abandono según nivel socioeconómico, género y región, como también algunos de los factores asociados con esta tasa.

Entre los antecedentes de investigaciones realizadas sobre este tema en la Argentina predominan los estudios que, de acuerdo a la primera perspectiva de análisis antes señalada, buscan determinar la dimensión y las causas del abandono en ciertas universidades o carreras (García de Fanelli, 2014a, Parrino, 2012). Una excepción es el trabajo de Landi y Giuliodori (2001), quienes calcularon la tasa de abandono global en 1998 para aquellos estudiantes que habían ingresado al sector universitario hasta el año 1984 a partir de una encuesta especial realizada en dicho año. Los resultados expuestos en este artículo buscan avanzar dentro de esta última línea de investigación, analizando además los factores asociados con la probabilidad de abandonar el sector universitario como un todo.

Comenzamos el artículo realizando una breve síntesis de los principales antecedentes teóricos en el estudio de los factores que inciden en el abandono universitario, principalmente centrándonos en la obra de Tinto (1975) y en los cambios experimentados por esta literatura en la última década. En particular, mostramos distintos enfoques dependiendo de si el plano de análisis estudiado es el abandono que tiene lugar en una universidad $O$, como es de interés en este trabajo, en el sector universitario como un todo. A continuación, exponemos cuál es el diseño y la metodología empleada en esta investigación, para después dar cuenta de los resultados alcanzados. En las conclusiones abordamos tanto los principales hallazgos de este estudio como también reflexionamos sobre las políticas públicas e institucionales que, de acuerdo con la experiencia internacional y nacional, podrían contribuir a mejorar la persistencia y la graduación en las universidades. 


\section{FACTORES ASOCIADOS CON EL ABANDONO GLOBAL E INSTITUCIONAL}

Desde la primera formulación del modelo de Tinto sobre el abandono universitario en las universidades americanas en 1975, hasta los últimos desarrollos dentro esta línea de investigación, se aprecian cambios que contribuyen a enriquecer el estudio de esta cuestión ${ }^{3}$.

En su modelo, Tinto (1975) privilegia el estudio de la interacción entre el individuo y la institución para explicar la decisión de los estudiantes de abandonar las instituciones de educación superior. Para hacerlo toma en cuenta, por un lado, la teoría de Durkheim sobre el suicidio y, por el otro, el análisis costo-beneficio de la teoría económica.

Durkheim sostenía que los individuos que no estaban suficientemente integrados en la sociedad tendían a suicidarse, ya sea porque sus valores no se correspondían con los de ésta o bien porque sus interacciones con el resto de sus miembros eran insuficientes (Durkheim, 1965). Al aplicar esta teoría al abandono universitario, Tinto estableció una diferencia entre el abandono voluntario de aquel que se produce porque los alumnos no alcanzan a satisfacer los requerimientos académicos de aprobación de los cursos. El abandono voluntario, como el caso del suicidio en Durkheim, tiene relación con la falta de integración del estudiante al sistema social de la universidad. Esto se expresa en un bajo nivel de interacción social con los pares, los profesores y en escasa participación en las actividades extracurriculares, así como en desacuerdos con los principales valores de la institución. En cambio, si lo que motiva el abandono son problemas de rendimiento en el aprendizaje, las fallas de integración al sistema académico provocan el abandono involuntario del estudiante al no cumplir con las condiciones para sostener su condición administrativa de alumno regular dentro de la institución. El grado de compromiso del estudiante con la meta de obtención del título universitario permite también predecir el abandono definitivo del transitorio por transferencia a otra institución. Un compromiso alto en el plano individual puede llevar a la transferencia a otra institución con mayores o menores demandas académicas.

En relación con la aplicación del modelo económico de análisis costo-beneficio en la explicación del abandono universitario, Tinto señala que los estudiantes que no se adaptan adecuadamente al sistema social o académico de la universidad sopesan además los beneficios económicos que le puede reportar concluir sus estudios con

\footnotetext{
3 Para un análisis detallado de los cambios conceptuales de mayor relevancia en la literatura sobre el abandono universitario en los últimos cuarenta años en los Estados Unidos, véase Cabrera et al. (2014).
} 
Abandono de los estudios universitarios: dimensión, factores asociados y desafíos para la política pública

los costos directos y de oportunidad que demanda estudiar y no destinar ese tiempo a la actividad laboral.

En un trabajo posterior, Tinto (1993) clasifica los enfoques teóricos sobre el abandono en "psicológicos", "sociológicos", "económicos", "organizacionales" e "interaccionales". El primero enfatiza el papel de las variables individuales en la explicación sobre el abandono universitario. Los tres siguientes (sociológico, económico y organizacional) ponen el acento en los factores del ambiente que inciden sobre esta decisión individual. Finalmente, el enfoque interaccional (propuesto por Tinto), integra tanto los factores individuales como los ambientales, focalizándose en la integración del estudiante en el ambiente académico y social de la universidad. El modelo parte entonces de la experiencia de una persona en el sistema académico y social, medida por su integración normativa y estructural. Se sostiene que esta experiencia modifica el objetivo y el compromiso institucional de modo tal de conducir a la persistencia o al abandono. Los individuos ingresan a la educación superior con una serie de atributos dados (género, raza, habilidad), experiencias educativas (promedio de nota del secundario, logros académicos y sociales) y perfil familiar (atributos de estatus social, clima de valores, expectativas). Estos factores inciden de modo directo o indirecto sobre los logros en la educación superior e influyen además sobre el grado de compromiso del estudiante con la meta de alcanzar un título y con la institución.

La teoría de Tinto resulta de utilidad especialmente cuando se analiza el abandono que tiene lugar dentro de una universidad en particular. Otra línea de investigación ha prestado atención al abandono que tiene lugar en el plano del sistema de educación superior en su totalidad o en su efecto opuesto, la persistencia. Este tipo de estudios permite arrojar luz sobre los factores académicos y económicos previos al ingreso y selección de una institución de educación superior por parte de los estudiantes, complejizando además el análisis al incorporar distinto tipo de instituciones (en los Estados Unidos los colleges de cuatro años y los community colleges de dos años) y de estudiantes (tradicionales y no tradicionales ${ }^{4}$ ). Este es el caso del estudio realizado por Cabrera y La Nasa (2000), quienes analizaron una cohorte de estudiantes del nivel medio superior desde 1982 a lo largo de once años. Dentro de esta cohorte y hasta el año 1993, 35 por ciento de los estudiantes de nivel medio habían alcanzado un título de grado. Del análisis del perfil socioeconómico de los estudiantes se arribó a

\footnotetext{
${ }^{4}$ Los estudiantes no tradicionales normalmente son aquellos que no cumplen con los siguientes requisitos: edad de 18 a 24 años, estado civil soltero/a, sin hijos, que ingresan inmediatamente tras finalizar la escuela media, que estudian a tiempo completo, que dependen financieramente de los padres y que viven en los campus universitarios (Cabrera et al., 2014).
} 
conclusiones de interés. Así, los estudiantes de bajo nivel socioeconómico tenían sólo 13 por ciento de probabilidad de graduarse en un plazo de 11 años frente a un 57 por ciento de los estudiantes de más alto perfil socioeconómico. No obstante, esta brecha de 44 por ciento que separa la probabilidad de graduarse entre los de menor y mayor nivel socioeconómico se reducía a 25 por ciento cuando se tomaba en cuenta otros factores. En tal sentido, obtener un título de grado dependían no sólo del nivel socioeconómico de los estudiantes de nivel medio sino también de los recursos académicos 5 , las aspiraciones por alcanzar un título, el tipo de institución de educación superior a la cual los estudiantes concurrían, el haber tomado cursos de matemáticas y ciencias en el nivel medio y el tener hijos mientras estudiaban en la educación superior (Cabrera y La Nasa, 2000).

Estas dos miradas, una más centrada en lo que ocurre dentro de las instituciones, dados ciertos atributos personales, académicos, familiares y socioeconómicos de los estudiantes, y otra que pone el foco precisamente en todas estas condiciones demográficas, educativas y socioeconómicas que preceden al ingreso de los jóvenes en la educación universitaria, se complementan entre sí, particularmente a la hora de diseñar políticas públicas e institucionales para mejorar la retención y la persistencia.

Las acciones que se desprenden del estudio del abandono universitario en el plano sistémico se corresponden, en primer lugar, con políticas de mejora de la calidad y la equidad en el nivel medio, de modo tal de evitar la segmentación de la experiencia educativa según el nivel socioeconómico, género o raza o capital cultural de los estudiantes y en el acceso a la educación superior. En segundo lugar, surge como muy relevante brindar ayuda económica a los estudiantes universitarios, a fin de compensar los costos directos de los estudios y, en particular el costo de oportunidad de estudiar para los sectores de menores ingresos.

En las universidades, las acciones institucionales que, según Tinto (2012) elevan la retención y en general la probabilidad de que los estudiantes se gradúen son aquellas que apuntan a (1) las expectativas: el gobierno de la institución y la gestión universitaria establecen claras expectativas respecto de lo que se espera de los estudiantes y los docentes también lo hacen en el espacio del aula; (2) el apoyo: se

\footnotetext{
5 Para medir empíricamente este concepto se confeccionó un índice que incluía múltiples variables vinculadas con el rendimiento académico del estudiante y la intensidad y calidad del currículo académico de la escuela media a la cual asistía el joven.
} 
brinda soporte en el plano académico, social y económico; (3) la valoración y la retroalimentación: se evalúa los logros alcanzados por los estudiantes y se les provee de modo continuo una devolución sobre estos resultados y (4) la participación activa: especialmente en el ámbito del aula. Entre las actividades institucionales que se mencionan para alcanzar estos objetivos se señalan programas que apuntan a la orientación del estudiante, el desarrollo de comunidades de aprendizaje, la incorporación de tutorías de pares y de docentes, la mejora de la relación docente-alumno e incentivos para promover adecuadamente esta interacción entre ambos, el impulso de programas de investigación con participación de los estudiantes, la inclusión de becas de trabajo dentro de la propia institución y la incorporación de cursos introductorios sobre campos disciplinarios con altas tasas de fracaso. De acuerdo con un estudio encargado por la Pell Institute (Engle y O'Brien, 2007), las acciones institucionales para reducir el abandono universitario más exitosas en los Estados Unidos han sido aquellas que han sido obligatorias, bien difundidas o que identifican con claridad quiénes requieren de apoyo, coordinadas centralmente, donde se involucra al personal docente y administrativo a través de incentivos claros y que son partes de un esfuerzo mayor de la institución para mejorar la experiencia educativa y los resultados que alcanzan los estudiantes.

En suma, la discusión teórica sobre el abandono universitario señala dos caminos posibles a emprender en el esclarecimiento de este complejo fenómeno: situarnos en el plano de una institución en particular o mirar al sector universitario o superior como un todo. A estas distintas miradas le corresponden también acciones diferentes desde el plano de la política. Los gobiernos pueden poner en marcha políticas que mejoren las condiciones académicas y económicas de los estudiantes que acceden a la educación superior e impulsar a través de la regulación de la calidad y el financiamiento acciones institucionales alineadas con el objetivo de mejorar en cantidad y calidad la graduación universitaria. Las instituciones están usualmente preocupadas por mejorar sus índices de graduación, aumentando la retención, pero sin prestar atención, como es lógico, a los impactos sistémicos de sus decisiones organizacionales.

En este artículo optamos por estudiar el abandono en el plano sistémico sobre la base de las fuentes de información disponibles en el caso argentino. 


\section{DISEÑO DE LA INVESTIGACIÓN Y METODOLOGÍA}

El estudio del abandono en el sector universitario argentino lo hemos realizado partiendo del indicador de tasa global de abandono, similar al status dropout rates que se emplea en Kaufman et al. (2004).

Así, entenderemos por tasa global de abandono la proporción de los jóvenes entre los 18 y los 30 años que han declarado en la Encuesta Permanente de Hogares que han asistido a la educación universitaria, pero que al momento de la encuesta ya no asistían más y no obtuvieron un título universitario. Esta tasa se calcula mediante la siguiente ecuación:

Tasa global de abandono $=$ [Desertores/ (Graduados +Desertores)] $\times 100$

Esta tasa es más baja que la que se calcula en el nivel micro de las instituciones universitarias por varias razones. En primer lugar, tal como lo señaló Tinto (1975), un grupo importante de estudiantes que abandona la universidad lo hace para continuar sus estudios en otra carrera 0 institución. Por tanto, constituyen desertores en una universidad en particular, pero pueden no serlo en el plano del sistema de educación superior como un todo. En otras palabras, una proporción del abandono voluntario e involuntario que se observa en el nivel de una organización universitaria desaparece en la estadística del sector en su conjunto. En segundo lugar, las tasas de graduación dentro de una institución se calculan teniendo presente una cohorte de ingresantes y bajo el supuesto de una cierta duración formal de los estudios. En la práctica, los estudiantes demoran mucho más tiempo en obtener el título universitario. Esto ocurre por problemas de integración en el sistema académico de la universidad, reflejados en bajo rendimiento académico, interrupciones momentáneas de los estudios atribuibles a motivos familiares o económicos, rigidez de los planes de estudio y actividad laboral en el contexto de una oferta horaria poco flexible. Al estudiar, como lo hacemos en este trabajo, lo que ocurre en un grupo etario amplio (entre los 18 y 30 años), es de esperar que la proporción de graduados aumente, disminuyendo la de aquellos que abandonan el sector universitario como un todo.

La fuente de datos utilizada es la Encuesta Permanente de Hogares (EPH). La EPH es una encuesta nacional trimestral representativa estadísticamente de la población urbana del país ${ }^{6}$, que

\footnotetext{
6 Argentina es un país altamente urbanizado. El 91 por ciento de su población (compuesta por unos 40 millones de habitantes en el 2010) era urbana. Posee además una alta concentración en el área Metropolitana, integrada por la Ciudad de Buenos
} 
Abandono de los estudios universitarios: dimensión, factores asociados y desafíos para la política pública

releva de modo sistemático y permanente características demográficas, educativas, laborales y socioeconómicas de la población.

La población de jóvenes a analizar comprende a todos aquellos que han asistido al sector universitario argentino dentro del grupo etario de 18 a 30 años. Cabe al respecto tener presente que en el 2013 este sector universitario estaba integrado por 121 instituciones, 57 de gestión estatal y 64 privadas. A pesar de la mayor presencia del sector privado en el plano institucional, ocho de cada diez jóvenes estudian en el sector universitario público. En particular, 54 por ciento de la matrícula de las universidades nacionales se concentra en sólo seis universidades, entre las cuales destaca la Universidad de Buenos Aires con poco más de 300 mil estudiantes (Ministerio de Educación, 2014)7.

Teniendo entonces como fuente la EPH, un primer objetivo de este trabajo ha sido calcular la tasa global de abandono universitario en la última década (2003-2013), distinguiendo además su variabilidad según género, grupo socioeconómico y región.

Como se aprecia en la literatura sobre el abandono universitario analizada en la sección previa, la condición socioeconómica del hogar del estudiante constituye un factor central que afecta las oportunidades, expectativas y compromisos de los jóvenes en pos de persistir en los estudios hasta obtener el título universitario. Partimos entonces del análisis de las tasas globales de abandono según el ingreso per cápita del hogar, considerando a éste como un indicador del nivel socioeconómico del estudiante.

Por su parte, la dimensión regional nos permitió además analizar indirectamente las brechas en torno a la calidad de la formación educativa en el nivel medio. Al respecto debemos tener presente que, de acuerdo a los resultados de la prueba PISA 2012, es posible apreciar importantes diferencias en los resultados de la Argentina según las

Aires y 24 partidos del Gran Buenos Aires (31,9 por ciento de la población) y la región Pampeana, compuesta por el resto de la provincia de Buenos Aires, Córdoba, Entre Ríos, La Pampa y Santa Fe (34,4 por ciento de la población) (INDEC, 2012). Cabe aclarar que el área Metropolitana o AMBA pertenece a la región Pampeana pero por su tamaño se la suele diferenciar en algunos análisis estadísticos mientras que en otros no se realiza esa diferencia.

7 En nuestra investigación no hemos tomado en cuenta al sector terciario no universitario que concentra aproximadamente al 30 por ciento de la matrícula de la educación superior. Este sector está integrado por más de dos mil pequeñas instituciones dedicadas a la formación docente en los distintos niveles de enseñanza y a la formación de técnicos (García de Fanelli, 2014b). Constituye un sector poco articulado con el universitario y provee títulos especialmente de pre-grado (equivalente a 5B en la clasificación CINE de la UNESCO). 
distintas regiones del país, obteniendo los mejores resultados la Ciudad de Buenos Aires (CABA) dentro de la región Pampeana (Albornoz y Warnes, 2013).

Estas brechas de calidad entre regiones también se reflejan en las pruebas de calidad que el Ministerio de Educación aplica a los estudiantes del último año de la escuela media. En este caso los resultados son aún más pertinentes para nuestro trabajo pues estos estudiantes constituyen los futuros ingresantes al sistema de educación superior (véase Tabla 1).

\begin{tabular}{|c|c|c|c|c|c|c|c|c|c|}
\hline \multirow[b]{2}{*}{ Nivel de Desempeño } & \multicolumn{3}{|c|}{ Lengua } & \multicolumn{3}{|c|}{ Matemática } & \multicolumn{3}{|c|}{ Ciencias Naturales } \\
\hline & Bajo & Medio & Alto & Bajo & Medio & Alto & Bajo & Medio & Alto \\
\hline Total Pais & 26,3 & 53,3 & 20,4 & 30,0 & 55,4 & 14,7 & 34,3 & 52,2 & 13,4 \\
\hline Región Pampeana & 22,7 & 52,7 & 24,6 & 25,0 & 56,6 & 18,4 & 31,4 & 53,1 & 15,5 \\
\hline Región Noreste & 38,7 & 51,7 & 9,6 & 47,7 & 48,4 & 3,9 & 47,8 & 45,6 & 6,6 \\
\hline Región Noroeste & 33,2 & 54,5 & 12,2 & 40,5 & 51,8 & 7,7 & 41,4 & 50,4 & 8,2 \\
\hline Región Cuyo & 26,8 & 55,0 & 18,2 & 30,2 & 57,7 & 12,2 & 33,3 & 53,7 & 13,1 \\
\hline Región Patagónica & 20,9 & 56,8 & 22,3 & 23,1 & 60,5 & 16,5 & 26,7 & 57,1 & 16,2 \\
\hline
\end{tabular}

En la Tabla 1 se puede apreciar, en primer lugar, que una alta proporción de los estudiantes del último año de la escuela media (en promedio uno de cada tres) obtiene bajos rendimientos en lengua, matemática y ciencias naturales. En segundo lugar, es clara la brecha entre regiones, alcanzando en la mayoría de los casos los mejores resultados los alumnos de la región pampeana ${ }^{8}$ y un desempeño más bajo los de las regiones norte del país, una de las más pobres en términos económicos.

En suma, el cálculo de la tasa global de abandono por región nos permite examinar en qué medida estas brechas socioeconómicas y de calidad en la oferta educativa se reflejan también en estas tasas.

Tras realizar este análisis descriptivo del abandono universitario en la Argentina, y teniendo como horizonte temporal la última década (2003-2013), 9 estudiamos los factores asociados con la tasa global de

\footnotetext{
${ }^{8}$ En este caso la región Pampeana incluye a la Ciudad de Buenos Aires y al Gran Buenos Aires.

9 La información analizada corresponde al III trimestre de cada año, a excepción del año 2007 en que se utilizó información correspondiente al II trimestre por no estar disponible en este caso el III trimestre.
} 
Abandono de los estudios universitarios: dimensión, factores asociados y desafíos para la política pública

abandono del sector universitario argentino por medio de un modelo de regresión logística ${ }^{10}$.

A través del modelo de regresión logística es posible indagar acerca de la importancia de ciertos factores que pueden incidir sobre la probabilidad de abandonar definitivamente los estudios universitarios. En función de las variables que surgen como relevantes de la literatura internacional expuesta en la sección previa y en el caso argentino en particular (García de Fanelli, 2014a), y de la disponibilidad de información que brinda la $\mathrm{EPH}$, hemos incluido en el modelo las variables de género, clima educativo del hogar, ingreso per cápita familiar, la región en la que vive el estudiante y su situación laboral. Estos factores constituyen algunas de las variables demográficas y socioeconómicas que condicionan la experiencia académica y social de los jóvenes en su acceso y permanencia en la educación superior. Para ello se estiman los parámetros de los siguientes modelos de regresiones logísticas:

$$
\begin{aligned}
& Y_{i}=\alpha+\beta X_{i}+i_{p c f_{i}}+\text { region }_{i}+\varepsilon_{i} \\
& Y_{i}=\alpha+\beta X_{i}+q_{i}+\text { region }_{i}+\varepsilon_{i}
\end{aligned}
$$

Donde $Y_{t}$ es la variable que se quiere explicar, es decir, la probabilidad de que el individuo abandone los estudios. Esta es una variable dicotómica, que tiene valor igual a uno si el individuo $i$ abandonó los estudios y cero si no los abandonó. $X_{i}$ representa aquellas variables observables referidas a las características del individuo que inciden en la probabilidad de abandonar los estudios. En nuestro estudio utilizamos información acerca del género, el clima educativo del hogarl1 y la situación laboral'2. La variable ipcfi representa el ingreso per cápita familiar y la variable regioni es dicotómica e indica la región de residencia del individuo, ya sea Gran Buenos Aires (GBA), Norte (que incluye tanto NOA como NEA), Cuyo-Patagónica o Pampeana (esta última es la omitida), y permite detectar diferencias en las probabilidades de abandono entre regiones. En la ecuación (3), reemplazamos ipcfi por $q_{i}$, que son variables dicotómicas que representan el quintil de ingreso per cápita familiar del individuo (Q1,

\footnotetext{
10 Han utilizado esta técnica econométrica para análisis similares en la Argentina entre otros Gasparini (2002) y Paz y Cid (2012).

11 La variable clima educativo del hogar se ha construido en base a los años de educación de los individuos mayores a 18 años que viven en el hogar. Así, se consideró a los hogares cuyos miembros mayores a 18 años tuvieran menos de 6 años de escolaridad promedio como hogares con bajo clima educativo; a aquellos con un promedio de años de escolaridad entre 7 y 11 , como hogares con un clima educativo medio y a los que tenían 12 o más años de escolaridad promedio, como hogares con un clima educativo elevado.

12 La condición laboral se construyó como una variable dicotómica con valor 1 si el individuo tenía trabajo y 0 si el individuo no estaba trabajando.
} 
Q2, Q3, Q4 y por último Q5 que es la omitida); y $\square$ i es el término de error específico de cada estudiante.

\section{RESULTADOS}

En esta sección comenzamos, en primer lugar, analizando el grado de equidad en la cobertura de educación universitaria en la Argentina para después calcular las tasas de abandono universitario global total y según nivel socioeconómico, género y región. En segundo lugar, presentamos los hallazgos que resultan de estimar el modelo de regresión logística, identificando algunos factores que se asocian con el riesgo de abandonar la educación universitaria.

\subsection{El abandono global universitario: tasas y variaciones según nivel socioeconómico, género y región}

De acuerdo con la base de datos de la EPH, la proporción de jóvenes de 18 a 30 años universitarios ${ }^{13}$ entre los años 2003 y 2013 se ha mantenido en una proporción de $26-28$ por ciento a lo largo del periodo, aumentando la escolarización del quintil de menores ingresos (es decir el I). La proporción de jóvenes que dentro de este grupo etario y quintil de menores ingresos asistía o asistió al sector universitario creció de 10 por ciento en el 2003 a 12 por ciento en el 2013, aunque presentando cierta disminución respecto del año 2012 (véase Tabla 2).

\begin{tabular}{|lrrrrrr|}
\hline Año $\backslash$ Quintil & Q1 & Q2 & Q3 & Q4 & Q5 & Total \\
\hline $\mathbf{2 0 0 3}$ & $10 \%$ & $18 \%$ & $28 \%$ & $42 \%$ & $57 \%$ & $\mathbf{2 7} \%$ \\
$\mathbf{2 0 0 4}$ & $7 \%$ & $18 \%$ & $30 \%$ & $41 \%$ & $61 \%$ & $\mathbf{2 8 \%}$ \\
$\mathbf{2 0 0 5}$ & $8 \%$ & $18 \%$ & $29 \%$ & $45 \%$ & $57 \%$ & $\mathbf{2 8 \%}$ \\
$\mathbf{2 0 0 6}$ & $9 \%$ & $16 \%$ & $31 \%$ & $42 \%$ & $56 \%$ & $\mathbf{2 8 \%}$ \\
$\mathbf{2 0 0 7}$ & $10 \%$ & $22 \%$ & $30 \%$ & $38 \%$ & $57 \%$ & $\mathbf{2 8 \%}$ \\
$\mathbf{2 0 0 8}$ & $10 \%$ & $20 \%$ & $30 \%$ & $37 \%$ & $54 \%$ & $\mathbf{2 7} \%$ \\
$\mathbf{2 0 0 9}$ & $11 \%$ & $20 \%$ & $28 \%$ & $36 \%$ & $55 \%$ & $\mathbf{2 7 \%}$ \\
$\mathbf{2 0 1 0}$ & $11 \%$ & $20 \%$ & $29 \%$ & $39 \%$ & $58 \%$ & $\mathbf{2 8 \%}$ \\
$\mathbf{2 0 1 1}$ & $14 \%$ & $22 \%$ & $24 \%$ & $38 \%$ & $55 \%$ & $\mathbf{2 7 \%}$ \\
$\mathbf{2 0 1 2}$ & $14 \%$ & $20 \%$ & $29 \%$ & $36 \%$ & $47 \%$ & $\mathbf{2 6 \%}$ \\
$\mathbf{2 0 1 3}$ & $12 \%$ & $22 \%$ & $29 \%$ & $37 \%$ & $52 \%$ & $\mathbf{2 7} \%$ \\
Promedio & & & & & & \\
del período & $\mathbf{1 1} \%$ & $\mathbf{2 0 \%}$ & $\mathbf{2 9} \%$ & $\mathbf{3 9} \%$ & $\mathbf{5 5} \%$ & $\mathbf{2 7} \%$ \\
\hline
\end{tabular}

Tabla $n^{\circ}$ 2. Argentina. Proporción de jóvenes universitarios de 18 a 30 años sobre el total de individuos de 18 a 30 años en cada hogar según el quintil de IPCF 2003-2013.

Fuente: Elaboración propia sobre la base a la EPH

\footnotetext{
13 Comprende a los jóvenes de 18 a 30 años que al momento de la encuesta asistían a la educación universitaria (estudiantes) o asistieron (graduados y desertores).
} 
Abandono de los estudios universitarios: dimensión, factores asociados y desafíos para la política pública

En la Tabla 2 también se aprecia que, de acuerdo con la escolarización universitaria promedio para el periodo 2003-2013, aquellos jóvenes que pertenecían al grupo de la población con mayores ingresos (el $\vee$ quintil) participaban cinco veces más que los de menores ingresos en la educación universitaria.

En lo que respecta a la tasa global de abandono en el año 2013, la Figura 1 muestra que esta ascendía a 37,7 por ciento entre los individuos de 18 a 30 . Se debe tener presente que esta tasa de abandono nos revela el umbral mínimo de deserción global pues en esa franja etaria 73 por ciento de los jóvenes todavía se encuentran estudiando. Si suponemos que el comportamiento de este grupo de estudiantes será similar a los que ya alcanzaron el título o abandonaron sus estudios, es de esperar que se mantenga el valor calculado. No obstante es probable que dentro de este grupo que aún estudiaba al momento de la encuesta se encuentren estudiantes no tradicionales, de edades superiores a los 25 años y que, por tener que destinar tiempo al trabajo y a la familia, son los que suelen tener tasas más bajas de graduación. En particular, en los datos analizados, el 21 por ciento de los jóvenes que todavía permanecían estudiando eran mayores de 26 años en el 2013. Si suponemos que la propensión a desertar es mayor en este grupo etario de estudiantes no tradicionales, es de esperar que la tasa final de abandono pueda llegar a ser cercana al 50 por ciento.

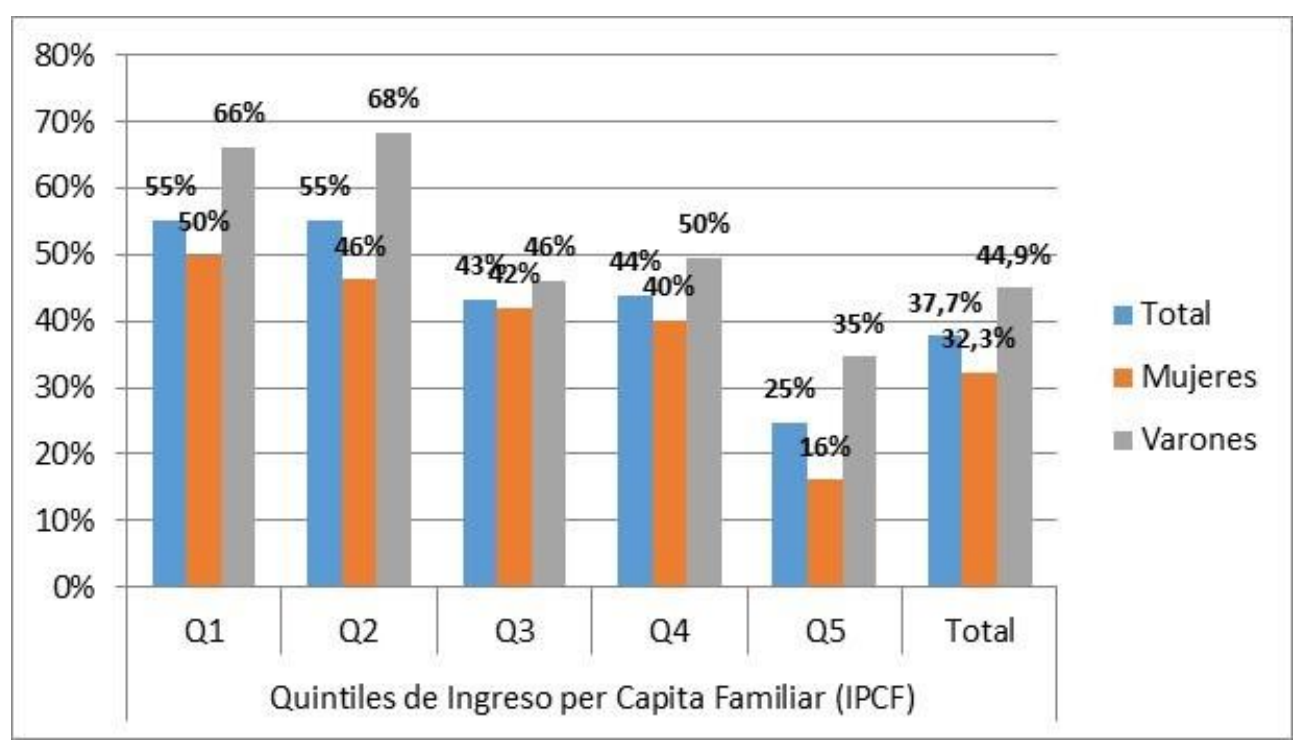

Figura $n^{\circ} 1$. Argentina. Tasa global de abandono por quintil de IPCF 2013 Fuente: Elaboración propia sobre la base de la EPH.

La tasa global de abandono que hemos calculado para un grupo etario es además más baja de lo que deja entrever las estadísticas parciales que aportan las universidades al Ministerio de Educación. Según estos datos, de cada 100 inscriptos en las universidades 
nacionales y privadas, egresan en promedio 28 estudiantes $^{14}$. Dentro del 72 por ciento restante se encuentran tanto los estudiantes que han abandonado en forma definitiva los estudios universitarios, especialmente a lo largo del primer año, los que interrumpieron momentáneamente su carrera para después de un tiempo continuar, los que cambiaron de carrera y/o institución y los que demoraron mucho más tiempo que el 50 por ciento por encima de la duración formal en obtener el título.

Al analizar la tasa global de abandono para el grupo etario de 18 a 30 años según el ingreso per cápita familiar (IPCF), se observa una diferencia sustancial entre el comportamiento de los individuos que pertenecen a los sectores de menores ingresos (Q1 y Q2) y el más alto (Q5). Estos datos revelan que el abandono es notablemente mayor entre los que pertenecen a los estratos de menor nivel socioeconómico y entre los varones (véase Figura 1).

Finalmente, al examinar la tasa global de abandono por región se observa que este indicador varía según la zona de residencia de los universitarios, siendo más baja en el área metropolitana del Gran Buenos Aires, integrada por la Capital Federal y 24 partidos que rodean a la Ciudad de Buenos Aires, y más alta en la región norte (región NOA y NEA), una de las regiones más pobres del país y con peores resultados en las pruebas de calidad aplicadas a los alumnos del quinto año del nivel medio (véase Figura 2 y Tabla 1).

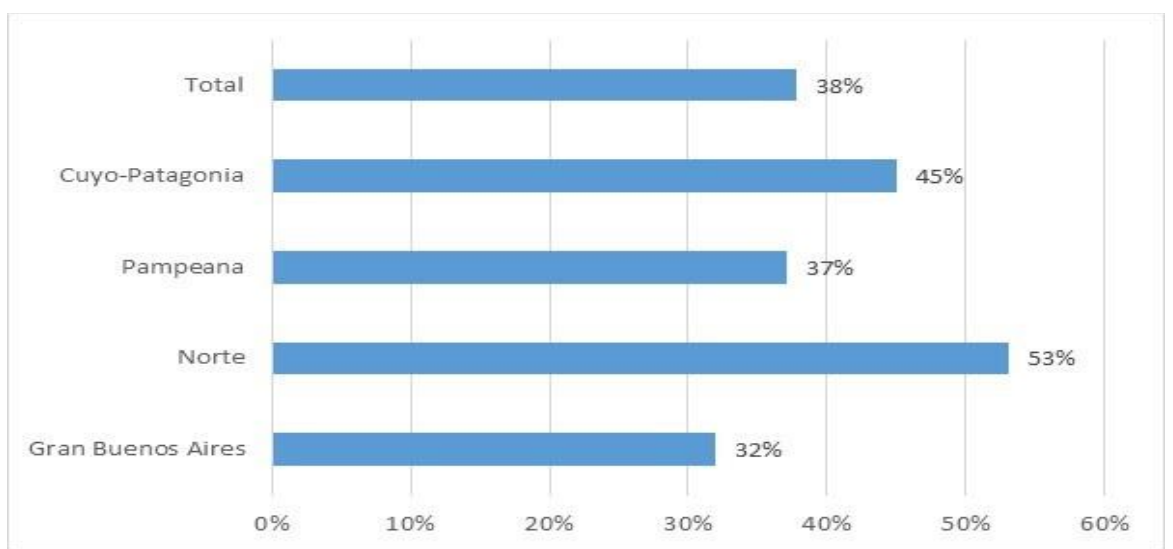

Figura $n^{\circ} 2$. Argentina. Tasas de abandono global por región de los jóvenes de 18 a 30 años, 2013. Fuente: Elaboración propia sobre la base de la EPH.

\footnotetext{
14 Para el cálculo se tomó en cuenta el promedio de egresados en las universidades públicas y privadas entre los años 2009 y 2011 y el promedio de los nuevos inscriptos entre los años 2002 y 2004 (Ministerio de Educación, 2014). En la Argentina, las carreras de grado suelen tener una duración de cinco años, pero también hay oferta de programas de cuatro años en el sector privado y carreras como ingeniería o medicina cuya duración puede ser de seis años. Por ese motivo, en el cálculo tuvimos en cuenta un periodo de siete años de estudios.
} 
Abandono de los estudios universitarios: dimensión, factores asociados y desafíos para la política pública

\subsection{Factores asociados con la probabilidad de abandonar la universidad}

Luego de haber brindado información sobre la magnitud del abandono, a continuación presentamos los resultados de las regresiones logísticas (ecuaciones (3) y (4)), que se utilizaron para poder determinar la incidencia de ciertos factores sobre la probabilidad de abandonar los estudios universitarios (véase Tabla 3).

\begin{tabular}{|c|c|c|c|c|c|c|}
\hline & $\begin{array}{c}\text { Razón de } \\
\text { Prob. }\end{array}$ & $\begin{array}{c}\text { Desvío } \\
\text { Estándar }\end{array}$ & $\begin{array}{c}\text { Estadíst. } \\
\mathbf{z}\end{array}$ & $\begin{array}{c}\text { Razón de } \\
\text { Prob. }\end{array}$ & $\begin{array}{c}\text { Desvío } \\
\text { Estándar }\end{array}$ & $\begin{array}{c}\text { Estadíst. } \\
\mathbf{z}\end{array}$ \\
\hline & \multicolumn{3}{|c|}{ (2) } & \multicolumn{3}{|c|}{ (3) } \\
\hline Género & 1,15 & 0,002 & $89,6 * * *$ & 1,15 & 0,002 & $87,1^{* * *}$ \\
\hline Clima Educativo del Hogar & 0,59 & 0,001 & $-264,1^{* * *}$ & 0,64 & 0,001 & $-215,1 * * *$ \\
\hline Condición laboral & 3,53 & 0,006 & $702,3 * * *$ & 3,87 & 0,007 & $721,0 * * *$ \\
\hline Ingreso Per cápita Familiar & 1,00 & 0,000 & $-241,2 * * *$ & & & \\
\hline Q1 & & & & 2,24 & 0,01 & $250,5 * * *$ \\
\hline Q2 & & & & 1,93 & 0,01 & $239,0 * * *$ \\
\hline Q3 & & & & 1,84 & 0,00 & $245,9 * * *$ \\
\hline Q4 & & & & 1,50 & 0,00 & $178,7^{* * *}$ \\
\hline Norte & 1,36 & 0,003 & $121,2 * * *$ & 1,29 & 0,003 & $99,2 * * *$ \\
\hline GBA & 1,06 & 0,002 & $28,2 * * *$ & 1,10 & 0,002 & $48,1^{* * *}$ \\
\hline Cuyo-Patagónica & 1,62 & 0,005 & $169,6 * * *$ & 1,61 & 0,005 & $167,1 * * *$ \\
\hline Cantidad de Observaciones & & & 15.796 .705 & & & 15.685 .624 \\
\hline LR chi2(9) & & & 687.104 & & & 715.759 \\
\hline Prob > chi 2 & & & & & & $\mathrm{c}$ \\
\hline Pseudo R2 & & & 0,0595 & & & 0,0625 \\
\hline
\end{tabular}

Tabla $n^{\circ} 3$. Factores que inciden en la probabilidad de haber abandonado los estudios universitarios en la Argentina. Fuente: Elaboración propia en base a información provista por la EPH de 2003 a 2013. Nota: ${ }^{* * *}$ indica un nivel de confianza estadística de $99 \%$.

En el caso de las regresiones logísticas, los coeficientes carecen de una interpretación directa. Por dicha razón, se ha optado por presentar la razón de probabilidades u odds ratio. Esta se define como la medida de asociación de que una condición, en este caso el abandono, se presente en un grupo de población frente al riesgo de que ocurra en otro. En el caso analizado, vemos por ejemplo el grado de asociación entre el género de la persona y la probabilidad de abandono. Así podemos observar que los varones tienen una probabilidad de abandonar los estudios universitarios 1.15 veces más grande que las mujeres. Asimismo, y tal como era esperable, cuanto mejor es el clima educativo del hogar menor es la probabilidad de que el individuo abandone los estudios. Ambos resultados se corresponden con los hallazgos de otros estudios realizados en la Argentina en la última década focalizados en el rendimiento académico de los estudiantes. Estos estudios muestran que el desempeño académico es mejor en el caso de las mujeres y de los jóvenes que provienen de 
hogares con mayor capital cultural (García de Fanelli, 2014a). Respecto de su condición laboral, el hecho que cuente con un trabajo está muy asociado con la probabilidad de haber abandonado (3,53 $\circ$ 3,87 modelos 2 y $3-$ ).

Por su parte, el ingreso per cápita del hogar, o el quintil al cual pertenece el estudiante, muestra que cuanto menor es el ingreso, mayor es la probabilidad de haber abandonado los estudios. Los individuos pertenecientes al primer quintil tienen una probabilidad 2,24 veces más grande de haber abandonado que aquellos pertenecientes al quinto quintil (este quintil es el que está omitido, y todos los estadísticos de los demás quintiles se refieren a la diferencia respecto de este). Así los que pertenecen al segundo quintil de ingreso per cápita familiar tienen una probabilidad de haber abandonado 1,93 veces mayor que los del quinto quintil.

En lo que respecta a las diferencias regionales, el hecho de pertenecer a la región Pampeana reduce las probabilidades de haber abandonado los estudios universitarios. Los individuos de todas las demás regiones tienen mayor probabilidad de haber abandonado los estudios que aquellos provenientes de esta región.

Finalmente, realizamos estimaciones del modelo por mínimos cuadrados ordinarios y se corroboró que todos los signos y la significatividad estadística son consistentes con los resultados obtenidos en la regresión logística (véase Anexo 1).

\section{CONCLUSIONES}

A partir del análisis realizado sobre la base de la Encuesta Permanente de Hogares se concluye que la proporción de jóvenes que han abandonado la educación universitaria en el 2013 en la Argentina asciende al 37,7 por ciento de aquellos que tenían al momento de la encuesta entre 18 y 30 años. Cuando se contrasta este valor con la tasa de graduación según datos aportados por cada universidad, se aprecia que sólo el 28 por ciento de los que ingresan en un determinado año, egresan en promedio siete años después. Este dato resulta muy preocupante para las universidades o las carreras que enfrentan problemas de retención, especialmente en el primer año. No obstante, lo que es percibido como un nivel de abandono alarmante al analizar las cifras institucionales o por carrera, pierde parcialmente la gravedad - sin dejar de constituir un problema significativo- cuando se calcula la tasa global de abandono. En efecto, es probable que una proporción importante de los estudiantes que ingresan en una universidad para estudiar una carrera dejen de hacerlo a lo largo del primer año por 
cuestiones que normalmente se las agrupa bajo el rótulo de "problemas vocacionales". Estos problemas comprenden tanto cuestiones de orden psicopedagógico, vinculadas con la difícil tarea de elegir a los 17 o 18 años la carrera académica o profesional a la cual le dedicarán el resto de sus vidas, como de falta de la información adecuada para tomar esta importante decisión. Por otro lado, otro grupo de estudiantes que son percibidos como desertores en las investigaciones realizadas dentro de las universidades, en realidad son jóvenes que no se han integrado adecuadamente al sistema social o académico de una universidad en particular, por lo cual han buscado otras alternativas institucionales que se ajusten mejor a su nivel de formación y a sus necesidades. Por último, en un sistema de educación universitaria que ya alcanzó el estadio de universalización, es esperable que el tipo de estudiante que comience a ganar presencia sea el no tradicional, es decir, jóvenes adultos que trabajan y estudian, que interrumpen momentáneamente sus carreras por motivos laborales o familiares y que por tanto demoran mucho más de lo esperado en obtener un título universitario.

Al analizar la tasa global de abandono de los jóvenes de 18 a 30 años también se observa que hay una importante brecha según sector socioeconómico, género y región de residencia. El 55 por ciento de los jóvenes que en el 2013 pertenecían a hogares de bajo ingreso per cápita había abandonado. Esta cifra se contrapone con el 25 por ciento en igual situación entre los de mayor ingreso per cápita. Por su parte, en igual año, el 45 por ciento de los varones desertaron de sus estudios frente al 32 por ciento de las mujeres. En términos regionales, se observa también variabilidad en la tasa global de abandono, presentando la tasa más baja la región del Gran Buenos Aires, donde se ubica la Ciudad de Buenos Aires y la más alta en la zona norte del país.

Al analizar el peso relativo de los distintos factores asociados con la probabilidad de abandonar los estudios universitarios, se confirma lo que señala la literatura respecto de la importancia del nivel socioeconómico, la actividad laboral y el clima educativo del hogar y el género. Asimismo resultó relevante la región de residencia del estudiante. La probabilidad de abandonar es mayor entre los varones, los que trabajan, los que habitan en hogares de menor ingreso per cápita familiar y nivel educativo y los que residen en las regiones norte del país.

Frente a estos resultados para el sector universitario en su conjunto, la política pública podría contribuir con la mejora en la persistencia y la equidad de resultados a través de tres acciones: (a) realizando encuestas específicas para indagar con mayor profundidad los factores que inciden sobre la tasa global de abandono universitario, (b) utilizando los mecanismos de aseguramiento de la calidad y de 
financiamiento de la oferta para incentivar y apoyar acciones institucionales que, en línea con lo que se señaló en Tinto (2012) y Engle y O'Brien (2007), busquen mejorar la integración social y académica de los estudiantes, especialmente de aquellos que provienen de hogares de menores recursos y con menor capital cultural y (c) ampliar el financiamiento destinado a ayuda económica para compensar las brechas de oportunidades existentes según en nivel de ingreso per cápita familiar de los estudiantes.

En la Argentina, en las últimas dos décadas, los gobiernos han promovido el desarrollo de programas de tutorías, especialmente de pares, y otras iniciativas para mejorar la articulación con el nivel medio. Asimismo se ha ampliado el número de becas disponibles para los sectores de menores recursos (García de Fanelli, 2014b). Sin embargo, al momento no existe información que permita corroborar la eficacia de estas políticas. No obstante, a la luz de la experiencia internacional, los principales desafíos que enfrentan para ser eficaces, no sólo en retener a los estudiantes sino también en aumentar la tasa de graduación, son aumentar la coordinación central de las iniciativas dispersas que se realizan en las instituciones, comprometer más activamente al conjunto de la comunidad universitaria en las acciones que se emprenden, generar incentivos económicos y simbólicos para aquellos que llevan adelante estas tareas, destinar una proporción mayor de fondos para aumentar el valor de las becas y de los programas en marcha e implementar políticas que atiendan la diversidad de situaciones en el plano regional.

\section{REFERENCIAS BIBLIOGRÁFICAS}

BOURDIEU, P. y PASSERON, J.C. (2004). Los herederos. Los estudiantes y la cultura. Buenos Aires: Siglo XXI.

BRUNNER, J. J. y FERRADA HURTADO, R. (2011) (eds.). Educación Superior en Iberoamérica. Informe 2011. Santiago de Chile: CINDAUNIVERSIA.

CABRERA, A. F. y LA NASA, S. (2000) Three Critical Tasks America's Disadvantaged Face on Their Path to College. New Directions for Institutional Research, 107, (23-29).

DOI: http://dx.doi.org/10.1002/ir.10702

CABRERA, A., PÉREZ MEJÍA, P. y LÓPEZ FERNÁNDEZ, L. (2014). Evolución de perspectivas en el estudio de la retención universitaria en los EEUU: bases conceptuales y puntos de inflexión. En FIGUERA, P. (ed.) Persistir con éxito en la universidad: de la investigación a la acción. Barcelona: Laertes.

DURKHEIM, E (1965 [1897]). El suicidio. Estudio de sociología. Buenos Aires: Schapire. 
Abandono de los estudios universitarios: dimensión, factores asociados y desafíos para la política pública

GARCIA DE FANELLI, A. (2014a). Rendimiento académico y abandono universitario: modelos, resultados y alcances de la producción académica en la Argentina. Revista Argentina de Educación Superior, Conocimiento y Difusión.6 (8) ,1-30.

(2014b). Educación superior en la Argentina: tendencias, políticas destacadas e incertidumbres. En Brunner, J.J. (ed.). Políticas de educación superior en Iberoamérica 2009-2013 (133-168). Santiago de Chile: Centro de Políticas Comparadas de la Universidad Diego Portales.

GASPARINI, L. (2002). On the Measurement of Unfairness: an application to high-school attendance in Argentina. Social Choice and Welfare, 19, 795-810. DOI: http://dx.doi.org/10.1007/s003550200156

LANDI, J. A. y GIULIDORI, R. (2001). Graduación y deserción en las universidades nacionales. En JOZAMI, A. y SÁNCHEZ MARTíNEZ, E. Estudiantes y Profesionales en la Argentina. Una mirada desde la Encuesta Permanente de Hogares (79-103). Buenos Aires: EDUNTREF.

MINISTERIO DE EDUCACIÓN (2014). Anuario 2011 de Estadística Universitaria. Buenos Aires: Ministerio de Educación.

OECD (2014). Education at a Glance 2014. Paris: OECD.

PARRINO, M. C. (2012). ¿Evasión o expulsión? Los mecanismos de deserción en el primer año universitario. (Tesis inédita de doctorado). Buenos Aires: Programa Interuniversitario Doctorado en Educación.

PAZ, J. A. y CID, J. C. (2012). Determinantes de la asistencia escolar de los jóvenes en la Argentina. Revista Electrónica de Investigación Educativa, 14 (1), 136-152.

TINTO, V. (1975). Dropout from Higher Education: A Theoretical Synthesis of Recent Research. Review of Educational Research, 45 (1), 89125. DOI: http://dx.doi.org/10.3102/00346543045001089

TINTO, V. (1993). Leaving College: Rethinking the Causes and Cures of Student Attrition. Chicago: University of Chicago Press.

TINTO, V. (2012). Completing College. Rethinking Institutional Action. Chicago: University of Chicago Press.

DOI: http://dx.doi.org/10.7208/chicago/9780226804545.001.0001

TROW, M. (2007). Reflections on the Transition from Elite to Mass to Universal Access: Forms and Phases of Higher Education in Modern Societies since WWII. En J. F. FOREST y P. G. Altbach (eds.) International Handbook of Higher Education (243-280). Vol. 18. Netherlands: Springer.

\section{FUENTES ELECTRÓNICAS}

ALBORNOZ, F. y WARNES, P. (2013). EI PISA y la desigualdad educativa en Argentina. [Consultado el 20 de enero de 2015], http://focoeconomico.org/2013/12/11/el-pisa-y-la-desigualdadeducativa-en-argentina/ 
DIRECCION NACIONAL DE INFORMACIÓN Y EVALUACIÓN DE LA CALIDAD EDUCATIVA (DINIECE). (2015). Operativo Nacional de Evaluación Calidad 2010. Censo de Finalización de la educación secundaria. Informe de Resultados. [Consultado el 30 de enero 2015],

http://repositorio.educacion.gov.ar:8080/dspace/bitstream/handl e/123456789/109660/Resultados\%20Censo\%200NE\%202010.pdf.

ENGLE, J. y O'BRIEN, C. (2007). Demography is not destiny: Increasing the graduation rates of low-income college students' at large public universities. Washington: The Pell Institute. [Consultado el 20 de noviembre de 2014], iles.eric.ed.gov/fulltext/ED497044.pdf.

INDEC. (2012). Censos Nacionales de Población y Vivienda, 2001-2010. [Consultado el 17 de enero de 2015], http://www.indec.mecon.ar/nivel2_default.asp?seccion=P\&id_te $\mathrm{ma}=2$.

INDEC (2003-2014) Encuesta Permanente de Hogares. [Consultado el 24 de enero de 2015], http://www.indec.mecon.ar/bases-dedatos.asp.

KAUFMAN, P., NAOMI, M. Y CHAPMAN, C.D. (2004). Dropout Rates in United States 2001. Washington: US Department of Education. [Consultado el 20 de enero de 2015], http://files.eric.ed.gov/fulltext/ED483073.pdf.

\section{ANEXO 1}

\begin{tabular}{|c|c|c|c|c|c|c|}
\hline & Coef. & $\begin{array}{l}\text { Desvío } \\
\text { Estándar }\end{array}$ & $\begin{array}{c}\text { Estadíst. } \\
\mathbf{t}\end{array}$ & Coef. & $\begin{array}{c}\text { Desvío } \\
\text { Estándar }\end{array}$ & $\begin{array}{c}\text { Estadíst. } \\
\mathbf{t}\end{array}$ \\
\hline & & (2) & & & (3) & \\
\hline Género & 0,02 & 0,0 & $93,3^{* * *}$ & 0,01 & 0,0 & $91,8^{* * *}$ \\
\hline Clima Educativo del Hogar & $-0,06$ & 0,0 & $-277,5 * * *$ & $-0,05$ & 0,0 & $-227,6 * * *$ \\
\hline Condición laboral & 0,12 & 0,0 & $740,0^{* * *}$ & 0,13 & 0,0 & $766,8^{* * *}$ \\
\hline Ingreso Per cápita Familiar & 0,00 & 0,0 & $-258,4 * * *$ & & & \\
\hline Q1 & & & & 0,08 & 0,0 & $244,9 * * *$ \\
\hline Q2 & & & & 0,07 & 0,0 & $247,6 * * *$ \\
\hline Q3 & & & & 0,07 & 0,0 & $264,3 * * *$ \\
\hline Q4 & & & & 0,04 & 0,0 & $196,1 * * *$ \\
\hline Norte & 0,03 & 0,0 & $114,4 * * *$ & 0,02 & 0,0 & $94,7^{* * *}$ \\
\hline GBA & 0,00 & 0,0 & $12,3 * * *$ & 0,01 & 0,0 & $36,3 * * *$ \\
\hline Cuyo-Patagónica & 0,05 & 0,0 & $168,0 * * *$ & 0,05 & 0,0 & $167,3 * * *$ \\
\hline Cantidad de Observaciones & & & 15.796 .705 & & & 15.685 .624 \\
\hline Prob $>F$ & & & - & & & - \\
\hline R2 & & & 0,042 & & & 0,044 \\
\hline Adjusted R2 & & & 0,0425 & & & 0,0449 \\
\hline
\end{tabular}

Tabla A.1 Factores que inciden en la probabilidad de haber abandonado los estudios universitarios en la Argentina (regresión por Mínimos Cuadrados Ordinarios). Fuente:

Elaboración propia en base a información provista por la EPH de 2003 a 2013. Nota: *** indica un nivel de confianza estadística de $99 \%$. 
Abandono de los estudios universitarios: dimensión, factores asociados y desafíos para la política pública

\section{Sobre las autoras:}

\section{Ana García de Fanelli}

Investigadora del Consejo Nacional de Investigaciones Científicas y Técnicas (CONICET) en el área de Educación Superior del CEDES (Centro de Estudios de Estado y Sociedad), centro de investigación asociado al CONICET en Buenos Aires, Argentina. Ha sido además Directora del CEDES entre los años 20082012. Es Licenciada y Doctora en Economía de la Universidad de Buenos Aires (UBA) y Magíster en Ciencias Sociales de la Facultad Latinoamericana de Ciencias Sociales (FLACSO). Como docente posee una extensa trayectoria en las Carrera de grado de Sociología y Economía de la Universidad de Buenos Aires y en el nivel de posgrado en las Universidades de Buenos Aires, Mar del Plata, Tres de Febrero y Cátedra UNESCO de la Universidad de Palermo. Sus líneas de investigación principales son el análisis comparado de las políticas de educación superior, el estudio de la universidad como organización y las políticas de financiamiento universitario. Ha sido consultora del IIPE-UNESCO Buenos Aires, IIPE-UNESCO París, CONEAU y CINDA. Ha publicado diversos libros, capítulos de libros y artículos en revistas especializadas nacionales y extranjeras.

Sitio Web: http://www.cedes.org/ana-maria-garcia-de-fanelli.php

\section{Cecilia Adrogué de Deane}

Profesora Asociada de la Universidad Austral, Argentina. Fue becaria postdoctoral del CONICET en la Universidad de San Andrés, visiting scholar del Center for International Higher Education de la Lynch School of Education en Boston College. Realizó los estudios correspondientes al Doctorado en Economía en la Universidad de San Andrés. Es Magíster en Economía de la Universidad del CEMA y es Licenciada en Economía de la Universidad Católica Argentina. Respecto de la labor de investigación, ha focalizado sus estudios en la economía de la educación. Entre otros, ha realizado estudios referidos a la calidad y equidad de la educación primaria pública, al efecto de los comedores escolares en los resultados académicos, al impacto de la doble jornada en la educación primaria y al desarrollo de la investigación en las universidades en respuesta a las políticas públicas para el aseguramiento de la calidad y financiamiento competitivo. Estos trabajos han sido publicados en libros, capítulos en libros y artículos en revistas científicas.

\section{Para citar este artículo:}

García, A. y Adrogué, C. (2015). Abandono de los estudios universitarios: dimensión, factores asociados y desafíos para la política pública. Revista Fuentes, 16, Junio, pp. 85-106. [Fecha de consulta: $\mathrm{dd} / \mathrm{mm} / \mathrm{aaaa}$. http://www.revistafuentes.es/

D.O.I.: http://dx.doi.org/10.12795/revistafuentes.2015.i16.04 